\title{
Perivascular Fat Density and Contrast Plaque Enhancement: Does a Correlation Exist?
}

(D) Saba, (D). Zucca, (D)A. Gupta, (D) Micheletti, (D).S. Suri, (D)A. Balestrieri, (D) M. Porcu, (D). Crivelli, (D) G. Lanzino, (D). Qi,

(D) V. Nardi, (D) G. Faa, and (D) R. Montisci

\begin{abstract}
BACKGROUND AND PURPOSE: Inflammatory changes in the fat tissue surrounding the coronary arteries have been associated with coronary artery disease and high-risk vulnerable plaques. Our aim was to investigate possible correlations between the presence and degree of perivascular fat density and a marker of vulnerable carotid plaque, namely contrast plaque enhancement on CTA.
\end{abstract}

MATERIALS AND METHODS: One-hundred patients (76 men, 24 women; mean age, 69 years) who underwent CT angiography for investigation of carotid artery stenosis were retrospectively analyzed. Contrast plaque enhancement and perivascular fat density were measured in 100 carotid arteries, and values were stratified according to symptomatic (ipsilateral-to-cerebrovascular symptoms)/asymptomatic status (carotid artery with the most severe degree of stenosis). Correlation coefficients (Pearson $\rho$ product moment) were calculated between the contrast plaque enhancement and perivascular fat density. The differences among the correlation $\rho$ values were calculated using the Fisher r-to-z transformation. Mann-Whitney analysis was also calculated to test differences between the groups.

RESULTS: There was a statistically significant positive correlation between contrast plaque enhancement and perivascular fat density ( $\rho$ value $=0.6582, P$ value $=.001)$. The correlation was stronger for symptomatic rather than asymptomatic patients $(\rho$ value $=$ $0.7052, P$ value $=.001$ versus $\rho$ value $=0.4092, P$ value $=.001$ ).

CONCLUSIONS: There was a positive association between perivascular fat density and contrast plaque enhancement on CTA. This correlation was stronger for symptomatic rather than asymptomatic patients. Our results suggest that perivascular fat density could be used as an indirect marker of plaque instability.

ABBREVIATIONS: $\mathrm{CPE}=$ contrast plaque enhancement; $\mathrm{PFD}=$ perivascular fat density

A bout one third proportion of ischemic strokes are caused by emboli from large-artery atherosclerosis usually involving the carotid artery. ${ }^{1-3}$ Traditionally, the degree of carotid stenosis is the main factor affecting therapeutic decisions. However, in recent years, similar to work in the coronary field, it has become clear that plaque composition

Received April 7, 2020; accepted after revision May 18.

From the Departments of Radiology (L.S., S.Z., G.M., A.B., M.P.), Pathology (G.F.), and Vascular Surgery (R.M.), Azienda Ospedaliero Universitaria, Monserrato (Cagliari), Italy; Department of Radiology (A.G.), Weill Cornell Medicine, New York, New York; Stroke Diagnosis and Monitoring Division (J.S.S.), AtheroPoint (TM), Roseville, California; Department of Radiology (P.C.), Azienda Ospedaliero Universitaria, Sassari, Italy; Department of Neurologic Surgery (G.L., V.N.), Mayo Clinic, Rochester, Minnesota; and Xuanwu Hospital (Y.Q.), Capital Medical University Beijing, China.

Please address correspondence to Luca Saba, MD, Department of Radiology, Azienda Ospedaliero Universitaria (A.O.U.), di Cagliari, Polo di Monserrato s.s. 554 Monserrato (Cagliari) 09045, Italy; e-mail: lucasaba@tiscali.it; @lucasabalTA

Indicates article with supplemental on-line table.

http://dx.doi.org/10.3174/ajnr.A6710 plays a very important role in the occurrence of cerebrovascular events. ${ }^{3-6}$

The different parameters associated with increased vulnerability include a lipid-rich necrotic core, ${ }^{7}$ intraplaque hemorrhage, ${ }^{8,9}$ and contrast plaque enhancement (CPE). ${ }^{10}$ In particular, CPE is a surrogate marker of microvessel density within the carotid plaque, and it is associated with plaque inflammation. ${ }^{11,12}$

Inflammatory changes in the fat tissue surrounding the coronary arteries have been associated with coronary artery disease and high-risk, ${ }^{13}$ vulnerable plaques, and in the past few years, some studies have investigated the perivascular fat density (PFD), measured as the density of pericoronary adipose tissue, as a parameter for coronary plaque evaluation. ${ }^{14,15}$ These articles showed that PFD was increased around culprit lesions compared with nonculprit lesions of patients with acute coronary syndrome. An increased PFD was recently described also in highrisk vulnerable carotid atherosclerotic plaque. ${ }^{16}$

In this study, we investigated the hypothesis that there is a correlation between the presence and degree of PFD and CPE. 


\section{MATERIALS AND METHODS}

\section{Study Design and Patient Population}

Institutional review board (University of Cagliari) approval was obtained, and informed consent was waived because of the retrospective nature of the study. To identify the minimum cohort size as having statistically significant data, we performed a power calculation (type I error, $\alpha=.10$; type II error, $\beta=0.15$; difference between correlation values $=0.2$ ) in which we estimated that a sample size of at least 93 carotid arteries would be sufficient to investigate the potential correlation between CPE and PFD. We further increased the number of carotid arteries, and we studied a total number of 100 arteries from 100 patients (76 men, 24 women; mean age, 70 years; age range, $46-87$ years).

Included were consecutive adult subjects who underwent CTA for suspected atherosclerotic disease of the carotid arteries from March 2016 to June 2017. Exclusion criteria included subjects younger than 18 years of age; CTAs performed for reasons other than suspected atherosclerotic disease (ie, dissection); and other etiologies for ischemic stroke such as evidence of a cardiac embolic source, evidence of an embolism from the thoracic aorta, and evidence of vertebrobasilar artery disease.

According to a standardized protocol, CTA of the carotid artery was performed for the following reasons: 1) Carotid sonography showed a significant stenosis $(>50 \%$ measured according to NASCET criteria) ${ }^{17}$ or features of plaque vulnerability (ulcerations, irregular surface), and 2) carotid sonography could not adequately assess the degree of stenosis and plaque characteristics because of anatomic conditions. Moreover, all subjects presenting with acute cerebrovascular events underwent CTA of the carotid arteries at the time of their acute CT of the head.

Part of the population $(n=56)$ of this study was included in previously published study on carotid plaque composition. ${ }^{18}$

\section{Classification of Cerebrovascular Symptoms}

In this study, both symptomatic and asymptomatic patients were considered, and only 1 carotid artery for each patient was included in the analysis. For asymptomatic patients, the carotid artery with the most severe degree of stenosis was included, whereas in symptomatic subjects, the carotid artery ipsilateral to the neurologic symptoms (eg, left hemispheric stroke, left carotid artery) was considered.

Patients were classified into symptomatic or asymptomatic as documented by the treating neurologist using the Trial of ORG 10172 in Acute Stroke Treatment (TOAST) criteria. ${ }^{19} \mathrm{We}$ considered as symptomatic a patient with a TIA or ischemic stroke in either cerebral hemisphere. TIA was regarded as a brief ( $<24$ hours) episode of neurologic dysfunction, such as hemiparesis, hemiparesthesia, dysarthria, dysphasia, or monocular blindness. If the episode of neurologic dysfunction exceeded 24 hours, it was classified as a stroke. We considered a lacunar stroke to have occurred when the patient showed one of the traditional clinical lacunar syndromes and did not have evidence of cerebral cortical dysfunction. Such patients also had normal CT/MR imaging findings or a relevant brain stem or subcortical hemispheric lesion with a diameter of $<0.5 \mathrm{~cm}$. The time window for inclusion in the symptomatic group was 6 months. We considered as asymptomatic a patient with no symptoms referable to the carotid artery or who had a remote ( $>6$ months) history of symptoms at the time of examination.

\section{CTA Technique}

CTA of the carotid arteries was performed with multiple scanner technologies (16-, 40-, and 128-detector row CT systems) according to a standardized protocol. None of the patients who underwent CTA of the carotid arteries had a medical history of cardiac output failure or contraindications to iodinated contrast media. Patients were placed in the supine position with the head tilted back to prevent dental artifacts. The coverage was from the aortic arch to the carotid siphon with a caudocranial direction, and examinations were performed before and after administration of contrast material. An angiographic phase was obtained with the administration of 50 $70 \mathrm{~mL}$ of prewarmed contrast medium (Ultravist 370, iopromide; Bayer HealthCare) injected with a flow rate of $4 \mathrm{~mL} / \mathrm{s}$. A bolustracking technique was used to calculate the correct timing of the scan. Dynamic monitoring scanning began 6 seconds after the beginning of the intravenous injection of contrast material. The trigger threshold inside the ROI was set at $+80 \mathrm{HU}$ above the baseline. The delay between acquisitions of each monitoring scan was 1 second. When the threshold was reached, the patient was instructed to hold his or her breath, and after an interval of 4 seconds, scanning started in the caudocranial direction. CT technical parameters were the following: section thickness $=0.6 \mathrm{~mm}$, interval $=0.3 \mathrm{~mm}$, matrix size $=512 \times 512$ pixels, FOV $=14-19 \mathrm{~cm}$, and application for reconstruction.

\section{Contrast Plaque Enhancement Analysis}

Hounsfield unit measurements were performed by 2 radiologists in consensus. Interactive window/level settings were usually set at $\mathrm{W}=850 \mathrm{HU}: \mathrm{L}=300 \mathrm{HU}$, progressing to very wide settings in the case of dense calcifications. ${ }^{20}$ First, the contrast material datasets were assessed in a circular or elliptic ROI ( $\geq 1 \mathrm{~mm}^{2}$; Fig 1) in the predominant area of the plaque to measure the Hounsfield unit values according to the method described by Saba et al. ${ }^{21}$ Areas showing contamination by contrast material or calcifications and regions of beam-hardening were excluded. In cases in which $50 \%$ of the plaque contained calcium components, we considered the plaque to be calcified, and this category of plaque was excluded. ${ }^{11}$

To obtain a correct registration between basal and postcontrast phases, radiologists visually assessed the corresponding slices when it was necessary to select another section along the $\mathrm{z}$-axis, the so-called matching process.

The section used was recorded to calculate the difference between the basal and contrast phase along the $\mathrm{z}$-axis. Following this matching phase, an ROI of the same area as that used in the contrast phase was put in the same position on the plaque to measure the basal Hounsfield unit value. The CPE is the difference in Hounsfield unit values obtained between the postcontrast and basal ROIs.

\section{Perivascular Fat Density Analysis}

The assessment of the PFD was performed by 2 radiologists in consensus 1 month later than the CPE quantification according to the approach described by Baradaran et al. ${ }^{16}$ The pixels 


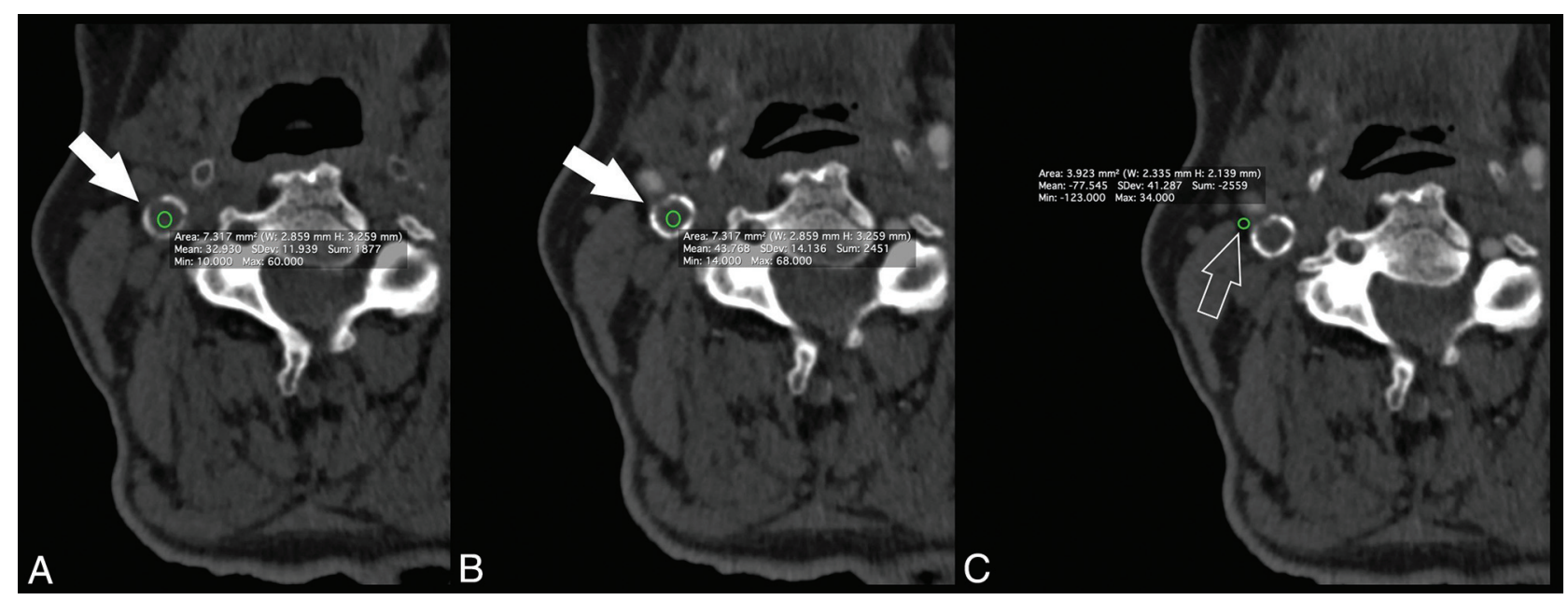

FIG 1. A 75-year-old man with right TIA. The basal scan (A) shows plaque (white arrow) of $32.93 \mathrm{HU}$, whereas the postcontrast scan (B) shows a plaque density (white arrow) value of $43.76 \mathrm{HU}$ with a CPE of $10.83 \mathrm{HU}$. The PFD (white open arrow, C) was $-77.545 \mathrm{HU}$.

Table 1: Demographics ( $n=100$ patients)

\begin{tabular}{lcccc}
\hline & $\begin{array}{c}\text { Complete Cohort } \\
(\boldsymbol{n}=100)\end{array}$ & $\begin{array}{c}\text { Symptomatic } \\
(\boldsymbol{n}=31)\end{array}$ & $\begin{array}{c}\text { Asymptomatic } \\
(\boldsymbol{n}=69)\end{array}$ & $\begin{array}{c}\boldsymbol{P} \\
\text { Value }\end{array}$ \\
\hline Age (yr) & 69 y (46-87) & 69.2 & 64.2 & .037 \\
Sex (male) & $76(76 \%)$ & $42(75 \%)$ & $34(77.2 \%)$ & .792 \\
Degree of stenosis & $61 \%$ & $67 \%$ & $53 \%$ & .001 \\
Hypertension & $45(45 \%)$ & $26(46 \%)$ & $19(44 \%)$ & .746 \\
Coronary artery disease & $49(49 \%)$ & $30(53.6 \%)$ & $19(43.4 \%)$ & .302 \\
Smoking status & $35(35 \%)$ & $23(41.1 \%)$ & $12(27 \%)$ & .151 \\
Diabetes & $23(23 \%)$ & $15(26.8 \%)$ & $8(18.2 \%)$ & .312 \\
Lipids & $59(59 \%)$ & $35(62.5 \%)$ & $24(54.6 \%)$ & .422 \\
\hline
\end{tabular}

\section{RESULTS}

\section{Baseline Characteristics}

Sixty-three patients had bilateral plaques, and 27 had unilateral plaques. Thirty-one patients were symptomatic for cerebrovascular symptoms, and 69, asymptomatic. Clinical and demographic characteristics are summarized in Table 1 . The average length of the plaque was $22 \pm 12 \mathrm{~mm}$, and the average maximum wall thickness was corresponding to adipose tissue were identified, and 2 ROIs (each $2.5 \mathrm{~mm}^{2}$ ) were placed in the perivascular fat present on the same axial section showing the maximal NASCET-defined ICA stenosis, $^{22}$ and the averaged values were considered.

The site of ROI placement was not exactly the same for each subject and was determined at the maximum stenosis site, location of the carotid plaque, and location of the perivascular fat pads. ROIs were drawn carefully to include only detectable fat density (visually dark and confirmed by negative Hounsfield units). Care was taken to exclude the carotid artery wall or surrounding soft-tissue structures, with ROIs placed at least $1 \mathrm{~mm}$ from the outer margin of the carotid artery wall. The process was re-checked before final acceptance.

\section{Statistical Analysis}

The normality of each continuous variable group was tested using the Kolmogorov-Smirnov Z-test. Continuous data were described as mean $\pm \mathrm{SD}$, and binary variables were summarized as count (percentage). Correlation coefficients (Pearson $\rho$ product moment) were calculated between the CPE and PFD. The differences among the correlation $\rho$ values were calculated using the Fisher r-to-z transformation. Mann-Whitney analysis was also calculated to test the differences between the groups. A $P$ value $<.05$ indicated statistical significance, and all correlation values were calculated using a 2-tailed significance level. $\mathrm{R}$ statistical and computing software (www.r-project.org) was used for statistical analyses.
$5.1 \pm 1.8 \mathrm{~mm}$. The average degree of stenosis was $61 \%$ (minimum, 3\%; maximum, 94\%). Thirty-nine carotid arteries had a NASCET stenosis of $>50 \%$ (mean degree of stenosis, $64 \%$; minimum, 50\%; maximum, 94\%). The summary values of the CPE and PFD are in the On-line Table.

\section{Carotid Plaque Enhancement and Perivascular Fat Density Correlation Analysis}

We found a positive correlation between CPE and PFD (Fig 2) ( $\rho$ value $=0.6582, P$ value $=.001)$. In the separate analysis of symptomatic and asymptomatic patients, symptomatic patients showed a stronger correlation compared with asymptomatic patients ( $\rho$ value $=0.7052, P$ value $=.001$ versus $\rho$ value $=0.4092, P$ value $=$ $.001)$.

In a second model of analysis, carotid plaques without CPE were excluded $(n=34)$, and a significant positive correlation between CPE and PFD was observed ( $\rho$ value $=0.7043, P$ value $=.001)($ Fig 2$)$. Similar to the first analysis, there was a stronger correlation in symptomatic than in asymptomatic patients $(\rho$ value $=0.6381, P$ value $=.001$ versus $\rho$ value $=$ $.5663, P$ value $=.001)$.

There was a significant difference in the $\rho$ values (CPE and PFD) between symptomatic and asymptomatic patients by considering the entire cohort of 100 patients (zeta statistic = -1.9904 ; $P$ value $=.0465)$. However, after excluding the 34 cases in which CPE was not detected, there was no significance in the $\rho$ values (zeta statistic $=-0.4312 ; P$ value $=.664$ ) . 

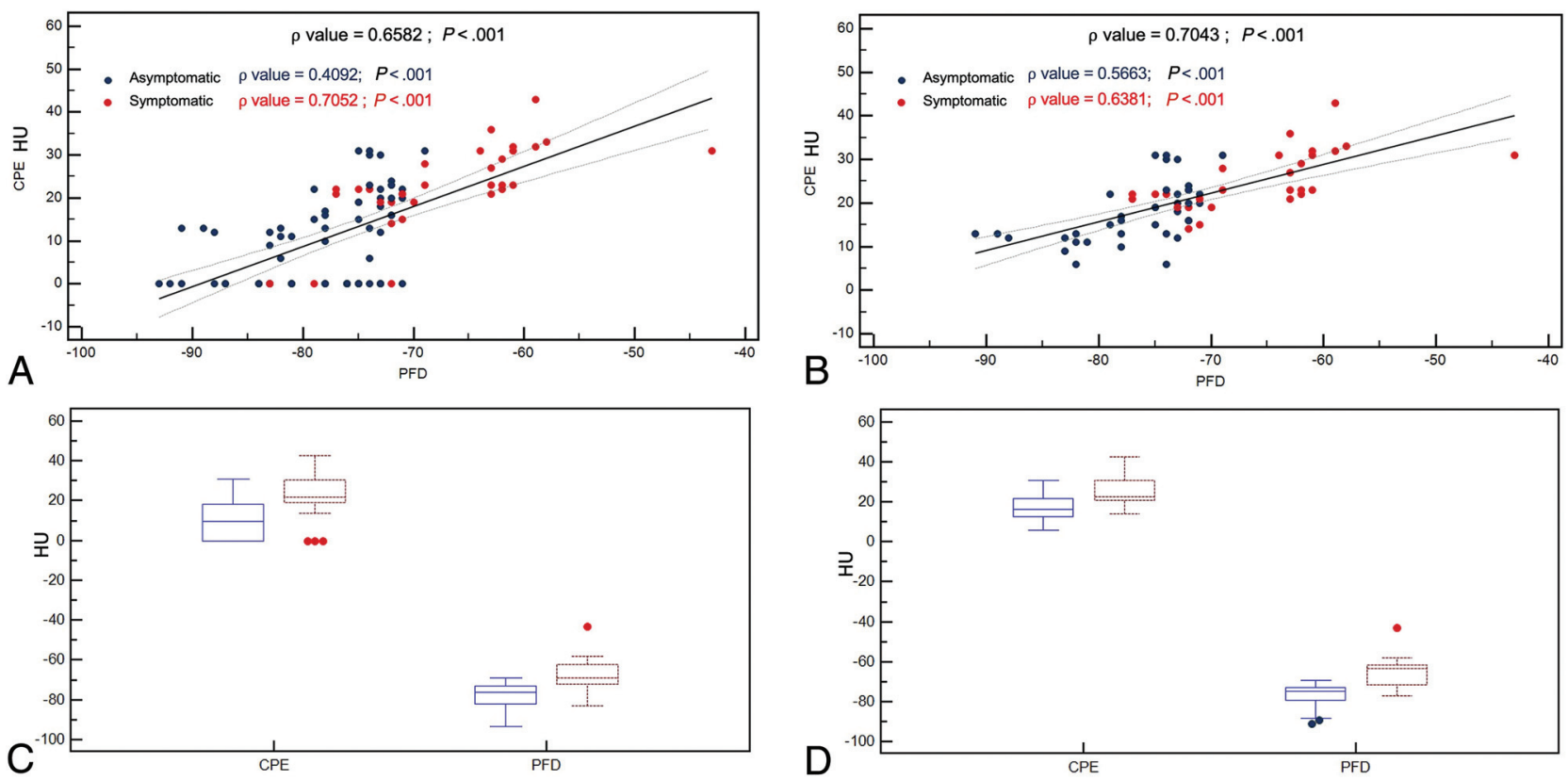

FIG 2. A, Scatterplot analysis with a regression line and $95 \%$ confidence intervals between the CPE and PFD with the asymptomatic (blue dots) and symptomatic patients (red dots) for the entire cohort of 100 patients. B, Scatterplot analysis with a regression line and $95 \%$ confidence intervals between the CPE and PFD with the asymptomatic (blue dots) and symptomatic patients (red dots) for the cohort of 66 patients who showed CPE. C, Boxplot plot analysis with $95 \%$ confidence intervals of CPE and PFD with the asymptomatic (blue lines) and symptomatic patients (red lines) for the entire cohort of 100 patients. D, Boxplot plot analysis with $95 \%$ confidence intervals of CPE and PFD with the asymptomatic (blue lines) and symptomatic patients (red lines) for the cohort of 66 patients who showed CPE.

Table 2: Mann-Whitney analysis for symptomatic-versus-asymptomatic patients

\begin{tabular}{lllll}
\hline & \multicolumn{2}{c}{ Cohort of 100 Patients } & \multicolumn{2}{c}{ 66 Patients with CPE } \\
\cline { 2 - 3 } & \multicolumn{1}{c}{ PFD } & CPE & PFD & CPE \\
\hline Average rank of first group & 38,971 & 40,7246 & 22,5263 & 25,6711 \\
Average rank of second group & 76,1613 & 72,2581 & 48,3929 & 44,125 \\
Mann-Whitney U & 274 & 395 & 115 & 234.5 \\
Test statistic Z (corrected for ties) & 5936 & 5122 & 5418 & 3865 \\
Two-tailed probability & $P<.001$ & $P<.001$ & $P<.001$ & $P=.001$ \\
\hline
\end{tabular}

Table 2 confirms a statistically significant difference in CPE and PFD according to the symptomatic or asymptomatic status of the patient.

\section{DISCUSSION}

In a cohort of 100 patients who underwent CTA for investigation of atherosclerotic carotid disease, we found a positive association between PFD and CPE on CTA. The correlation was stronger for symptomatic rather than asymptomatic patients.

In recent years, there has been growing interest in identifying features related to carotid plaque "instability," and several markers have been identified. ${ }^{3}$ Histopathologic studies have demonstrated that a common feature in vulnerable plaques is increased neovascularity and concomitant presence of inflammatory cells. These observations strongly suggest that one of the main pathways linked to plaque rupture is inflammation. ${ }^{23,24}$ Disruption of the plaque because of inflammatory and hemodynamic factors leads to ulceration and fissuring, which, in turn, result in loss of normal endothelium and luminal exposure of the necrotic lipid core. ${ }^{25}$ There is limited work on identification of objective markers of inflammation and instability in the carotid artery. Henrichot et al, ${ }^{13}$ in 2005 , in a histopathologic study, showed that inflammatory changes in the fat tissue surrounding coronary arteries are associated with coronary artery disease and high-risk plaques. Recently, such changes have been shown to be detectable on CT imaging in coronary $^{26}$ and carotid arteries. ${ }^{16}$

Inflammation and plaque neovascularization are 2 closely interrelated phenomena. ${ }^{27,28} \mathrm{CT}$ is reliable in the detection and grading of intraplaque neovascularization through CPE analysis. ${ }^{10,11,29}$ In our study, we investigated a correlation between the presence and degree of PFD and CPE. Indeed, we found a positive correlation between CPE and PFD, which, though present in both symptomatic and asymptomatic patients, was much stronger in symptomatic plaques $(\rho$ value $=0.7052, P$ value $=.001)$.

This finding confirms that pericarotid adipose tissue, which encases the carotid arteries without an intervening fascial barrier, is a metabolically and immunologically active fat deposit implicated in atherogenesis. The impact of the CT density was studied, in particular, for the association of cardiovascular events, and while a lower CT density of visceral and subcutaneous abdominal 
fat has been linked to a greater extent of cardiovascular events, ${ }^{30,31}$ in the Framingham Heart Study, ${ }^{31}$ the relationship between epicardial and paracardial fat density and high-risk plaque features is not well-understood.

Not all the carotid artery plaques show CPE, because intraplaque neovascularization can be absent depending on plaque type and characteristics. When we considered only carotid arteries showing CPE $(n=66)$, a statistically significant positive correlation between the CPE and PFD was maintained (Fig 2). After excluding plaques without $\mathrm{CPE}$, an even stronger correlation between $\mathrm{CPE}$ and PFD was found in symptomatic than in asymptomatic patients. ( $\rho$ value $=0.6381, P$ value $=.001$ versus $\rho$ value $=0.5663, P$ value $=.001)$.

The differences among the correlation $\rho$ values showed that the correlation between the CPE and PFD is not statistically stronger in symptomatic-versus-asymptomatic patients. This could be explained by the following:1) The low number (38 versus 28 patients) does not allow reaching the statistically significant threshold, or 2) those plaques without CPE represent a confounding factor because of the 34 plaques that did not show $\mathrm{CPE}$, only 3 cases were symptomatic.

We also found a statistically significant difference in CPE and PFD according to the symptomatic or asymptomatic status of the patient. These data are concordant with previous observations by Baradaran et $\mathrm{al}^{16}{ }^{16}$ who found that symptomatic patients had a higher mean PFD compared with asymptomatic patients $(-66.2 \pm 19.2$ versus $-77.1 \pm 20.4 \mathrm{HU}, P$ value $=.009)$, and by Saba et $\mathrm{al}^{11}{ }^{11}$ who found a statistically significant difference between symptomatic and asymptomatic patients for the presence of $\mathrm{CPE}(P$ value $=.001 ; \mathrm{OR}=7.5)$.

Our results are significant because they provide early validation of the use of PFD and/or CPE as noninvasive tools to assess inflammatory activity in carotid atherosclerotic disease. Further work is needed to understand the value of these biomarkers in predicting future cerebrovascular events or in identifying culprit plaques in patients with embolic strokes of undetermined sources. Furthermore, because PFD can be obtained on all CTA examinations while CPE requires both pre- and postcontrast imaging, future studies should aim to understand the incremental value of CPE above PFD alone, because in many centers, CTA is performed with a postcontrast acquisition alone. With further validation, these markers may be useful inclusion criteria for randomized trials incorporating anti-inflammatory stroke-prevention therapies in patients with carotid disease.

Our findings are concordant with ongoing research in the field of molecular imaging for the assessment of inflammation in atherosclerotic plaques, mainly performed with $\mathrm{PET} / \mathrm{CT}^{32,33}$ because of the potential to detect inflammation in plaques due to a high concentration of macrophages in such structures. ${ }^{34}$ Recent studies have shown that PET with $\left[{ }^{18} \mathrm{~F}\right] \mathrm{NaF}$ appears may be superior to $\left[{ }^{18} \mathrm{~F}\right]$ FDG in detecting the types of microcalcification that are markers of inflammation and high-risk plaques. ${ }^{35}$ Thus, PET techniques may serve as a valuable complement to more routinely and easily acquired CT to help clinicians detect vulnerable carotid plaques or so-called culprit plaques that have already given rise to an embolic event. Recent advances in translational research using
PET/CT have also enabled the visualization of perivascular inflammation in vulnerable atherosclerotic plaques. ${ }^{36,37}$

Our study has limitations. It is a retrospective study, and our data need confirmation in a larger cohort and should be considered as preliminary results. Although suggesting that PFD can be a surrogate of plaque instability, we cannot distinguish whether there is a causative relationship between the 2 or whether PFD is a mere epiphenomenon of the adjacent atherosclerotic process. Nevertheless, our data indicate, for the first time in the carotid artery, that an objective measure such as PFD can be of value as an indirect marker of plaque instability.

\section{CONCLUSIONS}

In patients with carotid artery stenosis investigated by CTA, there is a correlation between perivascular fat density and contrast plaque enhancement. This correlation is stronger for symptomatic rather than asymptomatic patients. Our results indicate that PFD could be used as an objective indirect marker of plaque instability in the carotid arteries.

Disclosures: Ajay Gupta_UNRELATED: Travel/Accommodations/Meeting Expenses Unrelated to Activities Listed: GE Healthcare and Siemens, Comments: reimbursement for travel expenses to site visits. Giuseppe Lanzino-UNRELATED: Board Membership: Superior Medical Experts, Nested Knowledge; OTHER RELATIONSHIPS: Marblehead Medical, LLC, Comments: shareholder (independently purchased shares).

\section{REFERENCES}

1. Gorelick PB. The global burden of stroke: persistent and disabling. Lancet Neurol 2019;18:417-18 CrossRef Medline

2. Kamel H, Navi BB, Merkler AE, et al. Reclassification of ischemic stroke etiological subtypes on the basis of high-risk nonstenosing carotid plaque. Stroke 2020;51:504-10 CrossRef Medline

3. Saba L, Saam T, Jäger HR, et al. Imaging biomarkers of vulnerable carotid plaques for stroke risk prediction and their potential clinical implications. Lancet Neurol 2019;4422:1-14 CrossRef Medline

4. Wasserman BA, Wityk RJ, Trout HH, et al. Low-grade carotid stenosis: looking beyond the lumen with MRI. Stroke 2005;36:2504-13 CrossRef Medline

5. Naghavi M, Libby P, Falk E, et al. From vulnerable plaque to vulnerable patient: a call for new definitions and risk assessment strategies: Part II. Circulation 2003;108:1772-78 CrossRef Medline

6. Saam T, Underhill HR, Chu B, et al. Prevalence of American Heart Association type VI carotid atherosclerotic lesions identified by magnetic resonance imaging for different levels of stenosis as measured by duplex ultrasound. $J$ Am Coll Cardiol 2008;51:1014-21 CrossRef Medline

7. Underhill HR, Hatsukami TS, Fayad ZA, et al. MRI of carotid atherosclerosis: clinical implications and future directions. Nat Rev Cardiol 2010;7:165-73 CrossRef Medline

8. Schindler A, Schinner R, Altaf N, et al. Prediction of stroke risk by detection of hemorrhage in carotid plaques. JACC Cardiovasc Imaging 2020;13(2 Pt 1):395-406 CrossRef Medline

9. Saba L, Micheletti G, Brinjikji W, et al. Carotid intraplaque-hemorrhage volume and its association with cerebrovascular events. AJNR Am J Neuroradiol 2019;40:1731-37 CrossRef Medline

10. Saba L, Lai ML, Montisci R, et al. Association between carotid plaque enhancement shown by multidetector CT angiography and histologically validated microvessel density. Eur Radiol 2012;22:2237-45 CrossRef Medline

11. Saba L, Mallarini G. Carotid plaque enhancement and symptom correlations: an evaluation by using multidetector row $\mathrm{CT}$ angiography. AJNR Am J Neuroradiol 2011;32:1919-25 CrossRef Medline 
12. Demeure F, Bouzin C, Roelants V, et al. Head-to-head comparison of inflammation and neovascularization in human carotid plaques: implications for the imaging of vulnerable plaques. Circ Cardiovasc Imaging 2017;10:e005846 CrossRef Medline

13. Henrichot E, Juge-Aubry CE, Pernin A, et al. Production of chemokines by perivascular adipose tissue: a role in the pathogenesis of atherosclerosis? Arterioscler Thromb Vasc Biol 2005;25:2594-99 CrossRef Medline

14. Marwan M, Hell M, Schuhbäck A, et al. CT attenuation of pericoronary adipose tissue in normal versus atherosclerotic coronary segments as defined by intravascular ultrasound. J Comput Assist Tomogr 2017;41:762-67 CrossRef Medline

15. Goeller M, Achenbach S, Cadet S, et al. Pericoronary adipose tissue computed tomography attenuation and high-risk plaque characteristics in acute coronary syndrome compared with stable coronary artery disease. JAMA Cardiol 2018;3:858-63 CrossRef Medline

16. Baradaran H, Myneni PK, Patel P, et al. Association between carotid artery perivascular fat density and cerebrovascular ischemic events. J Am Heart Assoc 2018;7:e010383 CrossRef Medline

17. Saba L, Mallarini G. Comparison between quantification methods of carotid artery stenosis and computed tomographic angiography. J Comput Assist Tomogr 2010;34:421-30 CrossRef Medline

18. Saba L, Lanzino G, Lucatelli $P$, et al. Carotid plaque CTA analysis in symptomatic subjects with bilateral intraparenchymal hemorrhage: a preliminary analysis. AJNR Am J Neuroradiol 2019;40:1538-45 CrossRef Medline

19. McArdle PF, Kittner SJ, Ay H, et al; NINDS SiGN Study. Agreement between TOAST and CCS ischemic stroke classification: the NINDS SiGN Study. Neurology 2014;83:1653-60 CrossRef Medline

20. Saba L, Mallarin G. Window settings for the study of calcified carotid plaques with multidetector CT angiography. AJNR Am J Neuroradiol 2009;30:1445-50 CrossRef Medline

21. Saba L, Mallarini G, Row M, et al. Carotid plaque enhancement and symptom correlations: an evaluation by using multidetector row CT angiography. AJNR Am J Neuroradiol 2011;32:1919-25 CrossRef Medline

22. Wardlaw J, Chappell F, Best J, et al. Non-invasive imaging compared with intra-arterial angiography in the diagnosis of symptomatic carotid stenosis: a meta-analysis. Lancet 2006;367:1503-12 CrossRef Medline

23. Pelisek J, Well G, Reeps C, et al. Neovascularization and angiogenic factors in advanced human carotid artery stenosis. Circ J 2012;76:1274-82 CrossRef Medline

24. Marnane M, Prendeville S, McDonnell C, et al. Plaque inflammation and unstable morphology are associated with early stroke recurrence in symptomatic carotid stenosis. Stroke 2014;45:801-06 CrossRef Medline
25. Steinberg D, Witztum JL. Lipoproteins and atherogenesis: current concepts. JAMA 1990;264:3047-52 Medline

26. Lu MT, Park J, Ghemigian K, et al. Epicardial and paracardial adipose tissue volume and attenuation: association with high-risk coronary plaque on computed tomographic angiography in the ROMICAT II trial. Atherosclerosis 2016;251:47-54 CrossRef Medline

27. Ammirati E, Moroni F, Magnoni M, et al. Circulating CD14+ and CD14highCD16 - classical monocytes are reduced in patients with signs of plaque neovascularization in the carotid artery. Atherosclerosis 2016;255:171-78 CrossRef Medline

28. Jaipersad AS, Shantsila A, Lip GY, et al. Expression of monocyte subsets and angiogenic markers in relation to carotid plaque neovascularization in patients with pre-existing coronary artery disease and carotid stenosis. Ann Med 2014;46:530-38 CrossRef Medline

29. Saba L, Tamponi E, Raz E, et al. Correlation between fissured fibrous cap and contrast enhancement: preliminary results with the use of CTA and histologic validation. AJNR Am J Neuroradiol 2014;35:754-59 CrossRef Medline

30. Alvey NJ, Pedley A, Rosenquist KJ, et al. Association of fat density with subclinical atherosclerosis. J Am Heart Assoc 2014;3:e000788 CrossRef Medline

31. Rosenquist KJ, Pedley A, Massaro JM, et al. Visceral and subcutaneous fat quality and cardiometabolic risk. JACC Cardiovasc Imaging 2013;6:762-71 CrossRef Medline

32. Fernández-Friera L, Fuster V, López-Melgar B, et al. Vascular inflammation in subclinical atherosclerosis detected by hybrid PET/MRI. J Am Coll Cardiol 2019;73:1371-82 CrossRef Medline

33. Fujimoto K, Norikane T, Yamamoto Y, et al. Association between carotid 18F-NaF and 18F-FDG uptake on PET/CT with ischemic vascular brain disease on MRI in patients with carotid artery disease. Ann Nucl Med 2019;33:907-15 CrossRef Medline

34. Chaker S, Al-Dasuqi K, Baradaran H, et al. Carotid plaque positron emission tomography imaging and cerebral ischemic disease: a systematic review and meta-analysis. Stroke 2019;50:2072-79 CrossRef Medline

35. Hop H, de Boer SA, Reijrink M, et al. 18F-sodium fluoride positron emission tomography assessed microcalcifications in culprit and non-culprit human carotid plaques. J Nucl Cardiol 2019;26:1064-75 CrossRef Medline

36. Mazurek T, Kobylecka M, Zielenkiewicz M, et al. PET/CT evaluation of 18F-FDG uptake in pericoronary adipose tissue in patients with stable coronary artery disease: independent predictor of atherosclerotic lesions' formation? J Nucl Cardiol 2017;24:1075-84 CrossRef Medline

37. Antonopoulos AS, Sanna F, Sabharwal N, et al. Detecting human coronary inflammation by imaging perivascular fat. Sci Transl Med 2017;9:eaal265 CrossRef Medline 